Decision-supporting models for human-reliability based safety promotion in offshore Liquid Natural Gas (LNG) terminal

Renyou Zhang ${ }^{\mathrm{a} *}$ and Henry Tan ${ }^{\mathrm{b}}$

${ }^{a}$ Department of Industrial Engineering, Tsinghua University, Beijing, China

${ }^{b}$ Lloyd's Register Foundation (LRF) Centre for Safety and Reliability Engineering,

School of Engineering, University of Aberdeen, Aberdeen, UK

*Corresponding author at: Department of Industrial Engineering, Tsinghua University,

Beijing, 100084, China. Email address: zhangry89@126.com (Renyou Zhang). 


\section{Decision-supporting models for human-reliability based safety promotion in offshore Liquid Natural Gas (LNG) terminal}

Offshore LNG terminal is a place which contains many high-risk operations. Although many protection instruments have been installed to guarantee the safety in offshore LNG terminal, many accidents still happen. Through the investigation of the accidents, people gradually realise that humans dominate the operational safety in offshore LNG terminal. Therefore, people need a human-reliability based decisionmaking model to make better plans for avoiding human errors. However, there is limited effort for reaching this requirement, so the particular research should be conducted. This study aims to separately introduce some learning algorithms such as neural networks and K-Nearest Neighbours (KNN) into the construction of the human-reliability based decision-supporting models. All these decision-supporting models will be based on the reality in the Beihai Offshore LNG Terminal. Then, the most robust model will be decided by the Mean Square Error (MSE) and the Average Error (AE) of each model.

Keywords: offshore LNG terminal; human-reliability based; decisionsupporting;

\section{Acronym}

AE: Average Error

ANFIS: Adaptive Neuro-Fuzzy Inference System

ANFIS-GP: Adaptive Neuro-Fuzzy Inference System Grid Partition

ANFIS-SC: Adaptive Neuro-Fuzzy Inference System Subtractive Clustering

ANN: Artificial Neural Network 
BPNN: Back Propagation Neural Network

KNN: K-Nearest Neighbours

LNG: Liquid Natural Gas

MCDM: Multi-Criteria Decision-Making

MSE: Mean Square Error

RBFNN: Radial Basis Function Neural Network

\section{Notation}

$\bar{d}$ : Centre data of Gaussian membership function

$d_{i}:$ Input data of neural network model

$M_{\text {avr }}$ : Average mark of all testing plans

$M_{p r e}$ : Predicted mark of each testing plan (generated by decision-supporting models)

$M_{\text {real }}$ : Real mark of each testing plan (generated by experts)

$S^{c}$ : The boundary value between acceptable condition and unacceptable condition

$S^{f}$ : The value after implementing the promoting method

$S^{i}$ : The initial value before implementing the new promotion plan

$S_{o}$ : The evaluated score for each attribute

\section{Introduction}

Shipping LNG currently takes up about over $50 \%$ of worldwide LNG transportation, so there is an increasing number of offshore LNG terminals constructed to receive and to store the LNG. With a large sum of LNG stored, these terminals become highly probably for fire and explosion. Besides, the environment around an offshore LNG terminal is harsh with the narrow working space, which decreases the human-reliability. Therefore, despite tremendous efforts on safety protection instruments supplied, still many tragedies happened during shipping LNG offloading process (Vanem et al., 2008), 
which negatively impacted people's health, assets, and even life. Through more indepth investigation, people gradually realised that loss prevention instruments are not sufficient to ensure the safety in LNG terminals since human errors are always the root reasons. Therefore, this paper aims to find a robust human-reliability based decisionsupporting system to guarantee the safety in offshore LNG terminal.

Nowadays, Artificial Neural Network (ANN) is widely used in addressing decisionmaking problems to replace the experts' judgements to avoid the arbitrary decision. This kind of method is a well-known artificial intelligence algorithm which is inspired by the biological phenomenon and has been developed for decades of years. Compared with many other decision-making methods, the ANN based techniques need fewer efforts in data analysis. So far, many remarkable achievements have occurred on neural networks (Makridaks, 2017). The ANN method effectively addresses the Multi-Criteria Decision-Making (MCDM) problem for supermarket supply chain choice (Golmohammadi, 2011; Özkan and İnal, 2014). The Adaptive Neuro-Fuzzy Inference System (ANFIS) model constructs a decision-supporting model for heart defect diagnosis (Sridevi and Nirmala, 2016). The ANFIS model also helps managers to evaluate the management plan and the working pressure for energy industry (Azadeh et al., 2016; Azadeh et al., 2013). The Back Propagation Neural Network (BPNN) allows managers in chemical plant to evaluate and to choose safety management plan (Guo et al., 2009). With such existing applications being successfully practised, it is meaningful to extend the concept to construct neural network models for a decision-supporting system for human-reliability based safety promotion plan's choice in offshore LNG terminal. 
Human-reliability is a considerable challenge to the rapidly increasing offshore LNG industry. According to historical data, over $50 \%$ of industrial accidents are fully or partly stimulated by human factors (Zhang and Tan, 2018). Besides, different from facility reliability which has sufficient statistical failure data, human-reliability always has limited data for use. Moreover, in the LNG industry, there is insufficient research on finding the human-reliability based safety plans to avoid human errors. Therefore, it is reasonable and meaningful to construct a supporting model for selecting the rational human-reliability promotion plan in offshore LNG terminal.

This study aims to construct a robust decision-supporting model and to determine the best human-reliability based safety promotion plan to avoid human errors for the Beihai Offshore LNG Terminal in China. The selected terminal contains four concrete LNG tanks with volume $160000 \mathrm{~m}^{3}$ each, so safety should be checked seriously. In this study, 63 human-reliability based safety promotion plans are collected from experienced experts. Different algorithms including several kinds of neural network models and KNN algorithm are applied to handle the decision-making process for the case "humanreliability based safety promotion plan's choice". Then, the performance of each method on MSE and AE are compared to identify the most reliable and robust approach. Finally, the $\mathrm{C}$ means method is utilised for classifying all 63 samples into three groups ("unacceptable", "acceptable", and "recommend"), so when making a decision, people not only can mark it but also can judge it. The arrangement of this paper is as follow. Section 2 explains the adopted methodology, and then in Section 3, the calculating process for Beihai Offshore LNG Terminal is presented by different approaches. In Section 4, the comparison of different methods is displayed and discussed to identify the best one. Finally, Section 5 gives the overall conclusion. 


\section{Adopted methodologies}

Many methods are available for building decision-making and supporting system. This study takes some ANN based techniques which include BPNN, Radial Basis Function Neural Network (RBFNN), ANFIS. Besides, the KNN method is also involved in constructing decision-making and supporting model. After identifying the most robust decision-supporting model, C means algorithm will be applied to distinguish all collected plans into three groups ("recommended", "acceptable", and "unacceptable").

The reasons for selecting the above-mentioned ANN based methods and KNN are presented below. First, all mentioned methods have already been applied for building decision-supporting models for other industries, so it should be suitable and meaningful for offshore LNG terminal also. Second, BPNN is a widely used and user-friendly method for model building and comparing. Third, RBFNN uses clustering methods to filter the input data in advance, so some useless neural nodes will not be triggered, and the time duration in data processing is reduced. Fourth, ANFIS, which connects fuzzy logic with neural network together to improve the results' quality, is currently a model that attracts increasing attention. Last, different from many ANN based methods, the procedures of $\mathrm{KNN}$ is crystal rather than a grey box. As a result, this research utilises the techniques mentioned above with the details illustrated as followings.

\section{BPNN}

The neural network algorithm is inspired by the current understanding of the biological neural system. It is a powerful tool that possesses the characteristics of self-learning, supervised learning, self-adaptive, linear, and nonlinear (Azadeh et al., 2008). Among a 
variety of neural network models, BPNN is a widely used method in many applications such as operational evaluation in the petrochemical plant (Guo et al., 2009), and electricity demand forecasting (Yang et al., 2016). A typical BPNN contains input layer (x1, x2, and x3 shown in Figure1), hidden layer, and output layer (f(x1 x2 x3) displayed in Figure 1). The BPNN consists of three main steps. Step 1 is the feedforward process for network training. Step 2 is the error evaluation process to calculate the error between the predicted data and the target data. Step 3 is the back forward propagation process to adjust the weight until the error in Step 2 is under tolerance level, and in this step, the gradient descent method is implemented to search the suitable weight data (Golmohammadi, 2011). Figure 1 gives the structure of BPNN.

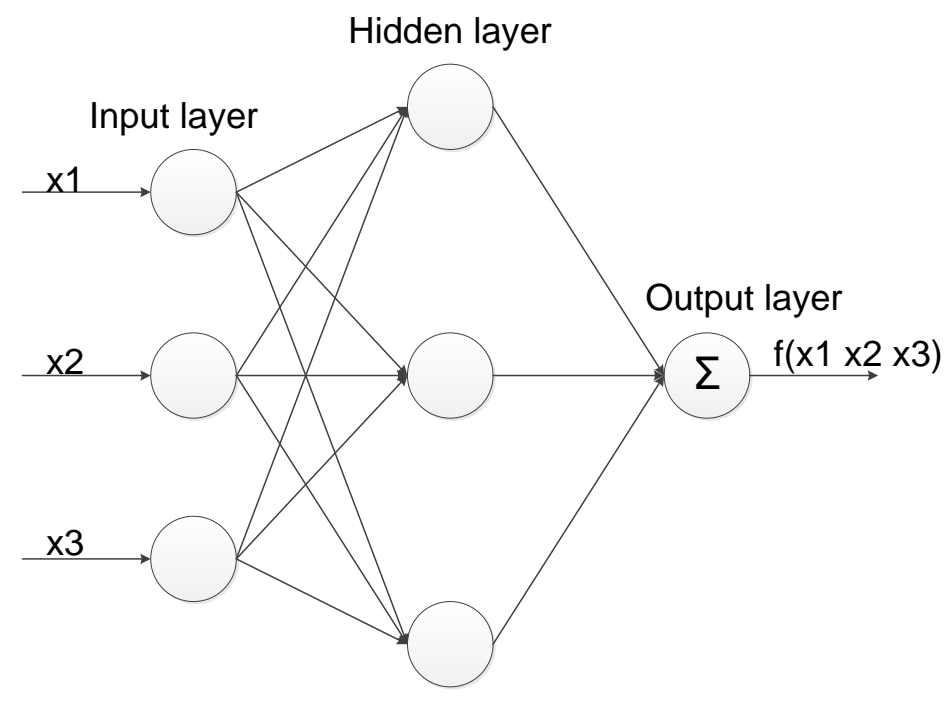

Figure 1. The structure of a BFNN structure.

\section{RBFNN}

The first application of radial basis function in constructing of neural network is from Broomhead and Lowe (Broomhead and Lowe, 1988). RBFNN has three layers. The first layer is made up of initial data shown as $\times 1 \times 2 \times 3$ in Figure 2. The second layer is the hidden layer which adopts radial basis function to transfer the source data, and the third layer is the output layer which is the summation of all outputs from the hidden layer 
(Ay and Kisi, 2014). Its final output value is expressed as $f(x 1 \times 2 \times 3)$ in Figure 2. The transformation from the input layer to the hidden layer is nonlinear, but the process from the hidden layer to the output layer is linear. During the transfer process from the input layer to the hidden layer, the most widely used activation function is Gaussian function. Besides, different from BPNN, RBFNN uses some clustering methods to identify all samples, so with clear identification of each sample, the training process is faster (Ay and Kisi, 2014). That is because, with precise identification of each sample, the irrelative neuron will not be activated. The model of RBFNN is shown in Figure 2.

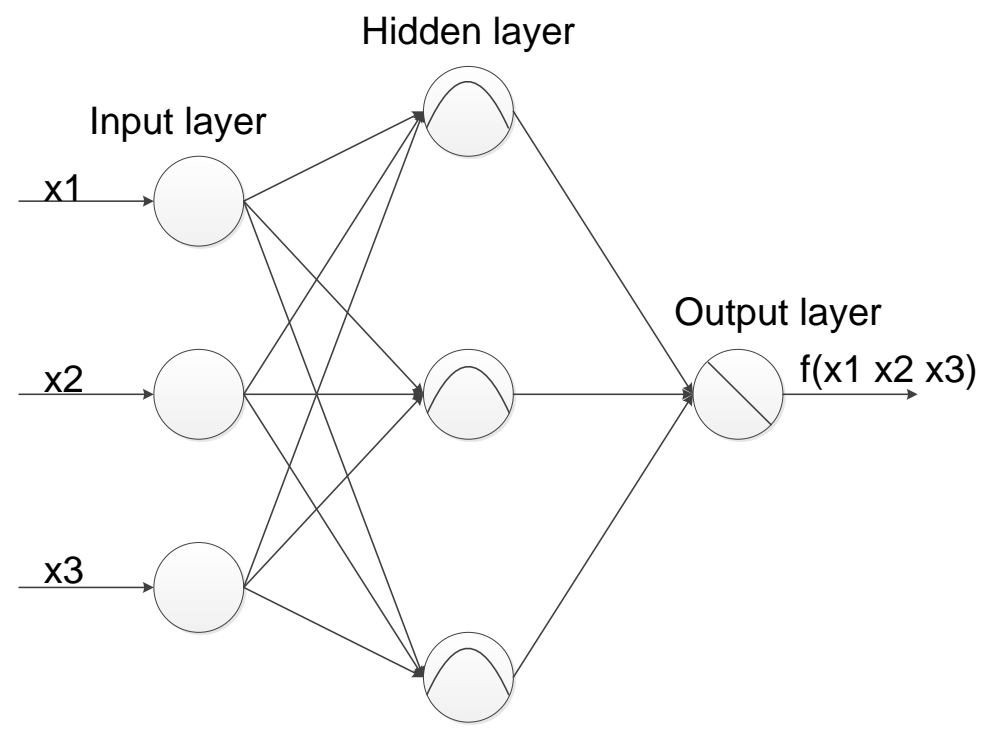

Figure 2. The structure of RBFNN.

\section{ANFIS}

ANFIS is a robust tool among neural network models. It combines fuzzy logic with neural network together to give simulation. As ANFIS accounts fuzzy logic, the structure of ANFIS is different from that of BPNN and RBFNN. Before the ANFIS process, a fuzzification process will be applied on source data to transfer them into the fuzzy membership degrees by fuzzy membership function. Then in the layer one, the fuzzy membership degree data will be introduced into ANFIS. In layer two, the "if- 
then" fuzzy logic rules will be put on all fuzzy membership degrees. Layer three will normalise the data that are deduced from layer two, and then in layer four, two consequence parameters will be introduced to identify the result of "if-then" rule. Finally, the fifth layer gives the final answer by the summation of all outputs from layer four, and this layer is known as the output layer. The gradient descent method is used to identify the parameters in fuzzy membership functions, and the least square method deals with the consequence parameters in layer four (Özkan and İnal, 2014). The ANFIS has two different procedures; one is Grid Partition (GP), the other is Subtractive Clustering (SC) (Wei, 2016). ANFIS-GP produces a Sugeno fuzzy inference system to deal with all data (Piotrowaski et al., 2015). ANFIS-SC not only can identify the fuzzy membership function but also can determine the number and locations of cluster centres among all data.

\section{KNN}

KNN algorithm is a very user-friendly supervised learning algorithm. It has been applied on data prediction (Xin and Chen, 2016), image processing, and pattern recognition (Sridevi and Nirmala, 2015). The letter " $\mathrm{K}$ " in KNN algorithm represents the number of the nearest samples that locate around the input sample. Usually, the Euclidean distance is adopted as the method to classify each input sample by evaluating the distance between the input sample and all other samples (Seetha et al., 2012). For instance, when $\mathrm{K}$ is three, three nearest samples around the input should be identified by Euclidean distance. If two nearest samples belong to group A and the rest one is group $\mathrm{B}$, then the input is group A. Then, the predicted output for the input sample is the mean value of the two corresponding outputs of the two nearest samples which are in same group with the input sample. 


\section{C means algorithm}

$\mathrm{C}$ means algorithm is one of the clustering techniques. It has already been applied in many practices such as image processing and disease diagnosis (Christ and Parvathi, 2011). Same with KNN algorithm, C means algorithm uses distance matrix also (Hartigan and Wong, 1979). The letter " $C$ " in this algorithm represents the number of clustering location centres (Ay and Kisi, 2014). The method starts with C (a real number) initially assumed clustering centres, and then to deduce the distance between the initially designed clustering centres and all other samples. Next, to identify the nearest clustering centre of each sample so that each sample can be temporarily assigned in the same group with its current nearest clustering centre. Afterwards, the new locations of clustering centres will be identified by calculating the mean value among all samples in each temporary cluster. Then, this procedure will repeat again and again until the new locations are same with previous locations. Finally, the centres of each cluster can be determined, and the group of each sample can be confirmed.

\section{Case study}

In the following study, the human-reliability based safety promotion plan's selection is applied to the Beihai Offshore LNG Terminal in China. 63 potential plans are collected and evaluated by the experienced experts. The ANFIS-GP, ANFIS-SC, BPNN, RBFNN, and $\mathrm{KNN}$ are introduced for constructing a decision-supporting model, and then their performances are compared. Here, three of all potential plans are listed below as the examples.

(1) Through investing more money in staff training to make sure they have full ability and knowledge to handle the situation safely and correctly. 
(2) Through recruiting higher qualified employee with enough expertise, and periodically reviewing the employee's working record performance to avoid and to correct the human errors in time.

(3) By appropriately increasing the number of operators to make sure their working capacity matches their workload.

\section{Data collection}

Facing these 63 suggested plans, evaluating and selecting the most suitable plan is necessary, and this is a Multi-Criteria Decision-Making (MCDM) problem. However, it always requires extensive efforts to express MCDM problem by mathematical equations, so this study firstly uses knowledge and experience based experts' judgements for evaluating each plan. During the MCDM process, economy is a significant factor (Borysiewicz et al., 2015), so the economic performance is involved in the MCDM process. Besides, the friendly time duration is beneficial to the practice of a plan, so it is a considerable factor for plan evaluation. In addition, since all plans are human-reliability based, the reliability performance of each plan should be involved as well. Therefore, in the plan evaluation process, we use "practicability", "economy", and "reliability" as the influence factors for final decision. Another challenge is, there is also no existing equation to directly calculate each influence factor, so Eq. (1) is adopted as the function to assess the performance score of each influence factor (Guo et al., 2009).

$$
S_{0}=\frac{S^{c}-S^{f}}{S^{c}-S^{i}}
$$

Where, $S_{o}$ is the evaluated score of each influence factor, $S^{c}$ is the boundary value between acceptable condition and unacceptable condition, $S^{f}$ is the value after adopting the specific plan, and $S^{i}$ is the initial value before implementing the new promotion plan. Therefore, based on the performance grade of each attribute collected by Eq. (1), 
experts can estimate the final mark of each potential plan. The evaluated scores of each influence factor and the corresponding final mark of each plan are listed as the Table A in the Appendix settled in the last part of this paper.

\section{Constructing the decision-supporting system}

Although the chosen experts have given the evaluation results for each plan, the process of experts' judgment is time-consuming and not economical. Therefore, it is possible to use the results determined by experts' judgements as the rule to construct the decisionsupporting model by some learning algorithms for the Beihai Offshore LNG Terminal. ANFIS-GP is firstly applied in this process to form the decision-supporting system. The evaluated scores of each influence factor (“practicability", "economy”, and, "reliability”) are viewed as the inputs for ANFIS-GP model, and the final marks which are given by experts are used as target outputs for ANFIS-GP. As fuzzy logic is involved in ANFIS, Gaussian membership function, which is displayed ad Eq. (2), is selected to represent each input attribute. For each input attribute, three levels ("low", "medium", and "high") are utilised to describe their performances.

$$
\operatorname{Gaussian}\left(d_{i}, \bar{d}, \sigma\right)=e^{-\frac{1}{2}\left(\frac{d_{i}-\bar{d}}{\sigma}\right)^{2}}
$$

Where, $d_{i}$ is the input data for ANFIS-GP model, $\bar{d}$ is the centre of Gaussian membership function, $\sigma$ is the width of Gaussian membership function. Among all provided data, this study takes $80 \%$ of all as the training set for ANFIS-GP simulating, and the rest $20 \%$ of all samples, which are labelled by "t" in the Table A of the Appendix, are used as the testing set to check whether the ANFIS-GP model is accurate enough. Then, taking these training data into ANFIS-GP model, and after 1000 iterations, the decision-supporting system is formed. As $20 \%$ of all samples are used as the testing set, taking them into ANFIS-GP model, the predicted results can be 
generated. Figure 3 is produced to display the comparison between the ANFIS-GP prediction and the real results from experts' judgement in the testing set.

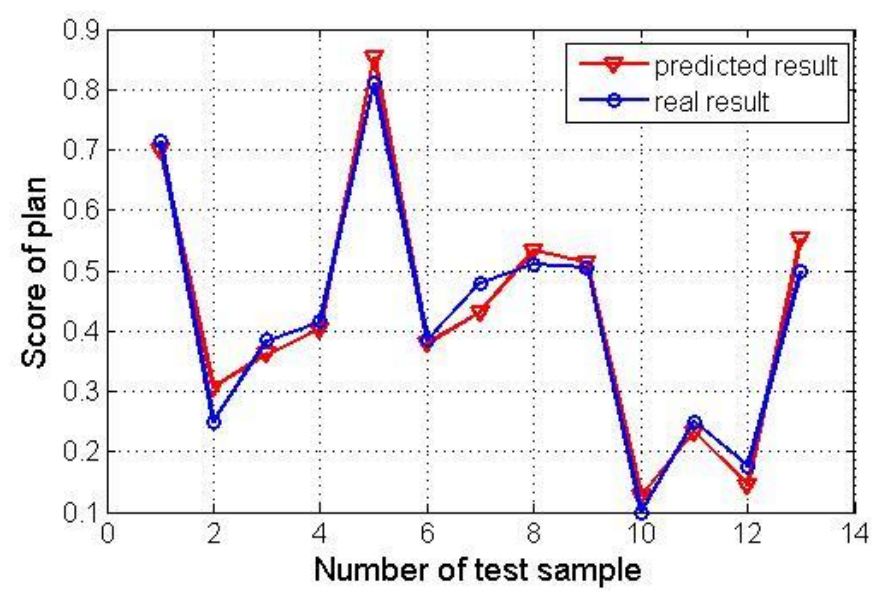

Figure 3. Comparison between the ANFIS-GP results and the real results from experts' judgement in the testing set.

Similar with ANFIS-GP, other four chosen methods (BPNN, KNN, RBFNN, and ANFIS-SC) are separately implemented in this study. The training set and the testing set are same as those of ANFIS-SC ( $80 \%$ for training and $20 \%$ for testing). The comparison between the results predicted by different models and the experts' evaluating results are shown in Figure 4. Table 1 concludes the real output data determined by experts and the estimated output data from all selected models.

(a)

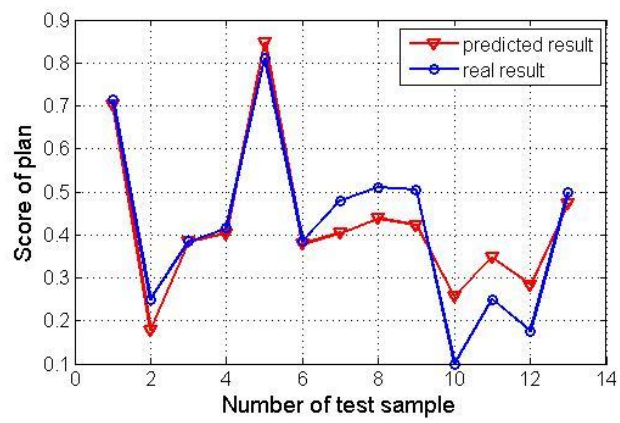

(b)

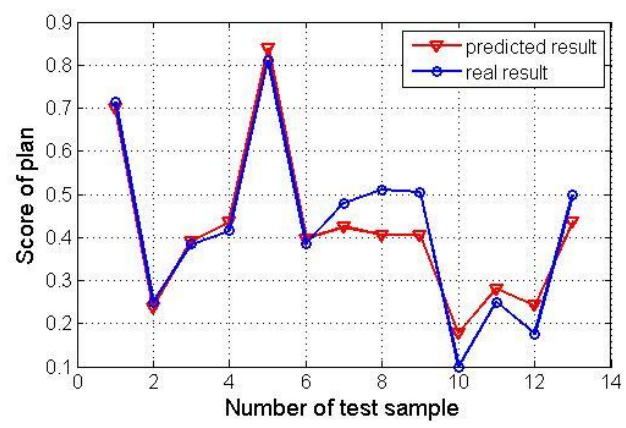


(c)

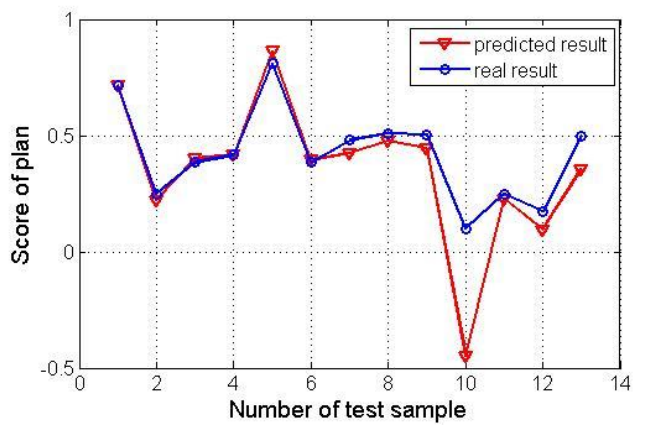

(d)

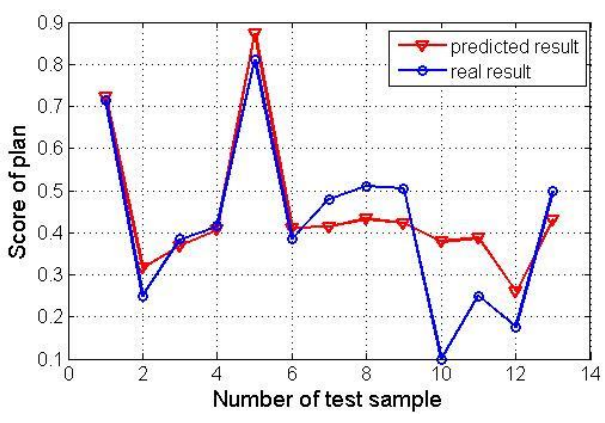

Figure 4. (a) Comparison between the BPNN predicted results and the real results. (b)

Comparison between the KNN predicted results and the real results. (c) Comparison between the RBFNN predicted results and the real results. (d) Comparison between the ANFIS-SC predicted results and the real results.

Table 1. The real experts' evaluating outputs in the testing set and all model based predicted outputs.

\begin{tabular}{|c|c|c|c|c|c|c|}
\hline No. & Real & $\begin{array}{c}\text { ANFIS- } \\
\text { GP }\end{array}$ & $\begin{array}{c}\text { ANFIS- } \\
\text { SC }\end{array}$ & $\mathrm{KNN}$ & BP-NN & RBFNN \\
\hline 1 & 0.715 & 0.700 & 0.722 & 0.700 & 0.702 & 0.718 \\
\hline 2 & 0.25 & 0.307 & 0.316 & 0.239 & 0.177 & 0.222 \\
\hline 3 & 0.385 & 0.362 & 0.369 & 0.392 & 0.384 & 0.400 \\
\hline 4 & 0.415 & 0.403 & 0.407 & 0.436 & 0.404 & 0.417 \\
\hline 5 & 0.810 & 0.854 & 0.873 & 0.838 & 0.849 & 0.865 \\
\hline 6 & 0.385 & 0.379 & 0.412 & 0.397 & 0.379 & 0.396 \\
\hline 7 & 0.480 & 0.431 & 0.414 & 0.424 & 0.404 & 0.423 \\
\hline 8 & 0.510 & 0.534 & 0.432 & 0.405 & 0.439 & 0.477 \\
\hline 9 & 0.505 & 0.513 & 0.422 & 0.405 & 0.422 & 0.445 \\
\hline 10 & 0.100 & 0.126 & 0.379 & 0.177 & 0.257 & -0.450 \\
\hline 11 & 0.250 & 0.232 & 0.387 & 0.281 & 0.347 & 0.226 \\
\hline
\end{tabular}




\begin{tabular}{rrrrrrr}
\hline 12 & 0.195 & 0.144 & 0.259 & 0.242 & 0.284 & 0.0940 \\
13 & 0.485 & 0.553 & 0.431 & 0.437 & 0.472 & 0.354 \\
\hline
\end{tabular}

\section{Performance comparison of each method}

From the calculation above, it seems all five approaches are capable of building a decision-supporting system for the safety plan's selection in the Beihai Offshore LNG Terminal, so identifying the most robust method is necessary. For the sake of finding the most robust model, MSE and AE are selected to evaluate each model. That is because MSE represents the distance between the result produced by decisionsupporting model and the real result assessed by experts, and AE indicates the deviation among a group of data. The smaller data of MSE and AE represent the better performance of the model. The definition of MSE and AE are expressed by Eq. (3) and (4). Their results for the testing set are shown as Table 2.

$$
\begin{gathered}
M S E=\frac{1}{n} \sum_{i=1}^{n}\left(M_{\text {pre }}-M_{\text {real }}\right)^{2} \\
A E=\frac{1}{n}\left(\sum_{i=1}^{n}\left|M_{\text {pre }}-M_{\text {avr }}\right|\right)
\end{gathered}
$$

where, $M_{\text {pre }}$ is the predicted final mark of all testing plan (generated by decisionsupporting models), $M_{\text {real }}$ is the real mark (generated by experts), and $M_{\text {avr }}$ is the average mark of all testing plans.

Table 2. Performance of ANFIS-GP, ANFIS-SC, BP-NN, RBFNN, and KNN.

\begin{tabular}{cccccc}
\hline Performance & ANFIS-GP & ANFIS-SC & BP-NN & RBFNN & KNN \\
\hline MSE & 0.00134 & 0.01 & 0.00514 & 0.0264 & 0.00287 \\
AE & 0.0308 & 0.0729 & 0.056 & 0.082 & 0.0429 \\
\hline
\end{tabular}

Therefore, from the information in Table 2, ANFIS-GP shows the best performance. Besides, as the ANFIS-GP can automatically adjust the input fuzzy membership 
functions, the membership functions of three input attributes and the fuzzy surface between each two of three attributes and ANFIS-GP output are shown as Figure 5 and Figure 6.
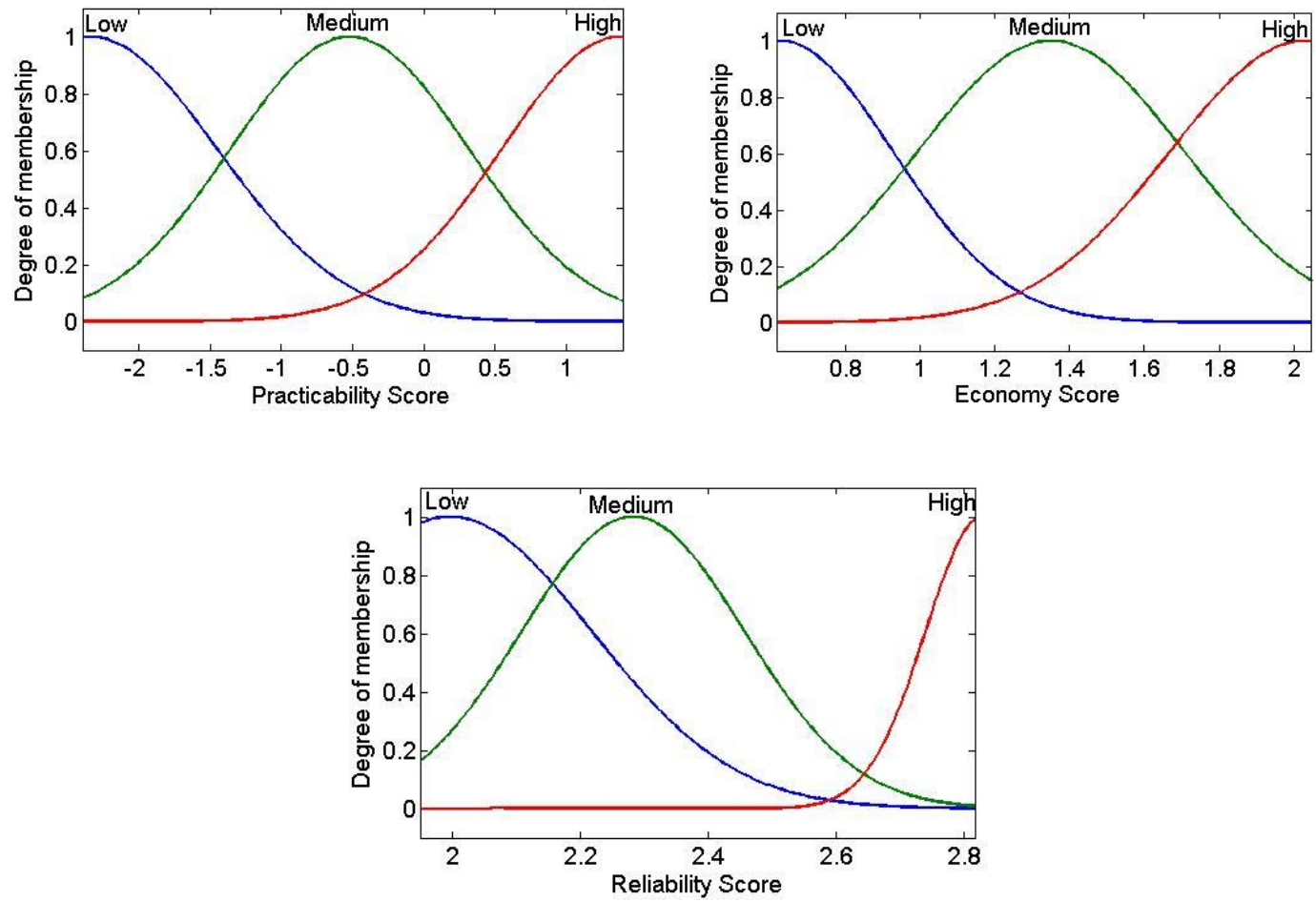

Figure 5. Adjusted membership functions for each influence factors.
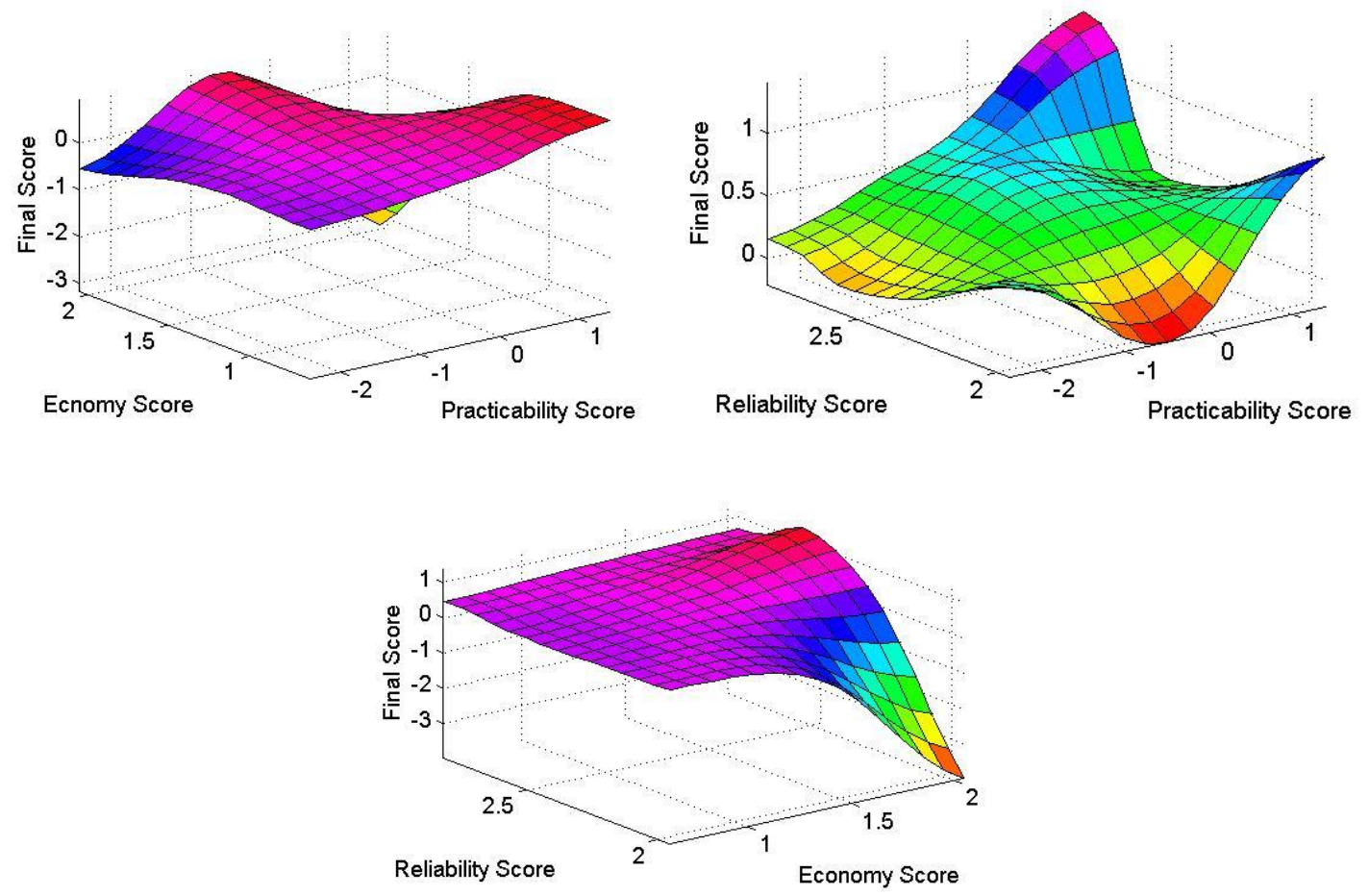
Figure 6. Fuzzy surfaces after training.

The decision-supporting system has been constructed, so when taking the input data into this system, the output results can be generated automatically without experts' efforts. However, only with the result from ANFIS-GP, it is still unclear for people to determine whether the plan is recommended or not since there is no clear standard. Therefore, to explicitly recognise whether the chosen plan is positive or negative is meaningful for decision-choice. The clustering method $\mathrm{C}$ means algorithm is a good option, so it is implemented to classify the group of recommended plan, the group of acceptable plan, and the group of unacceptable plan. Then, with the clustering results, people can clearly recognise the suitable plan. Three levels (Unacceptable, Acceptable, Recommend) are set for all plans in this study, so the parameter $\mathrm{C}$ in $\mathrm{C}$ means clustering can be set as "Three", which means there are three centres, one for unacceptable group, one for acceptable group, and one for recommended group. Then, applying $\mathrm{C}$ means clustering method on all evaluation results in the last column of the Table A in the Appendix, the three centres of $\mathrm{C}$ means approach are located at 0.2336 (Unacceptable group), 0.4496 (Acceptable group), and 0.7671 (Recommend group). Afterwards, with the centres of each group and the final scores of each sample (shown as the last column in Table A), the fuzzy membership function can be estimated. Gaussian membership function is utilised again to express each group. The centre of Gaussian membership function $(\bar{d})$ is the clustering centre which has been deduced by $\mathrm{C}$ means clustering, and the width of the Gaussian membership curve $(\sigma)$ is determined by Eq. (5).

$$
\sigma=\sqrt{\frac{1}{N} \sum_{i=1}^{N}\left(d_{i}-\bar{d}\right)^{2}}
$$

Where $N$ represents the number of the samples in the corresponding cluster. With the centre data and width data for Gaussian membership function, the fuzzy membership 
function curves for the final score of each potential plan are displayed as Figure 7. So when using the ANFIS-GP model to estimate the performance mark of the suggested plan, the membership level (Recommend, Acceptable, and Unacceptable) of the plan can be determined at the same time by checking the membership function curves in Figure 7. Finally, a solid decision can be made.

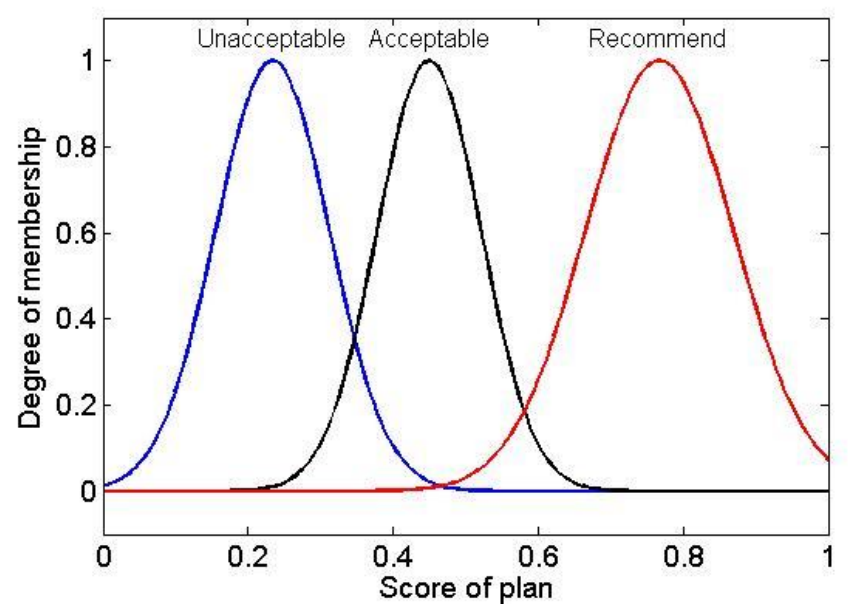

Figure 7. Fuzzy membership curves for the final results of each plan.

\section{Conclusion}

This paper constructs human-reliability based decision-supporting model for the safety plan's selection in the Beihai Offshore LNG Terminal. The model can be used for current and future rational decision-making and updating without experts' efforts. Through the comparison among all chosen methods, ANFIS-GP provides the best performance with MSE 0.00134 and AE 0.0308. Besides, according to the analysing results of 63 potential safety plans, the most suitable plan for this offshore LNG terminal is to increase the time for the safety inspection and maintenance work reasonably. Furthermore, it is worthy to note that, this decision-supporting model may also be suitable for safety promotion plans' choice in other industries, such as nuclear, commercial shipping, and chemical process industry. 


\section{Acknowledgement}

We would like to give great thanks to the experienced engineers in the Beihai Offshore LNG Terminal for their helpful support during the preparation of this paper. We would also like to express thanks to the editor and the anonymous reviewer for the valuable comments.

\section{Appendix}

Table A. Score of each plan.

\begin{tabular}{|c|c|c|c|c|}
\hline $\begin{array}{l}\text { Plan } \\
\text { No. }\end{array}$ & Practicability & Economy & Reliability & Final mark \\
\hline 1 & 1.395 & 0.948 & 2.667 & 0.950 \\
\hline 2 & -0.753 & 1.065 & 1.952 & 0.260 \\
\hline 3 & 1.000 & 1.350 & 1.952 & 0.830 \\
\hline 4 & -1.185 & 1.020 & 2.700 & 0.350 \\
\hline 5 & -1.830 & 2.048 & 2.743 & 0.305 \\
\hline 6 & 0.345 & 1.053 & 2.700 & 0.745 \\
\hline 7 & -1.244 & 2.021 & 2.690 & 0.450 \\
\hline 8 & 0.345 & 0.703 & 2.687 & 0.685 \\
\hline 9 & -1.435 & 0.897 & 2.467 & 0.200 \\
\hline 10 & -1.435 & 0.870 & 2.696 & 0.235 \\
\hline 11 & 0.541 & 0.940 & 2.620 & 0.735 \\
\hline 12 & -1.451 & 1.698 & 2.328 & 0.285 \\
\hline 13 & -1.247 & 1.668 & 2.769 & 0.375 \\
\hline 14 & 0.344 & 0.797 & 2.231 & 0.550 \\
\hline
\end{tabular}




\begin{tabular}{|c|c|c|c|c|}
\hline 15 & -1.846 & 1.506 & 2.336 & 0.145 \\
\hline 16 & -1.640 & 1.479 & 2.810 & 0.315 \\
\hline 17 & 0.345 & 0.825 & 2.675 & 0.700 \\
\hline 18 & -1.435 & 1.646 & 2.213 & 0.290 \\
\hline 19 & -1.001 & 1.619 & 2.817 & 0.455 \\
\hline 20 & -0.427 & 0.893 & 1.972 & 0.385 \\
\hline 21 & 0.542 & 0.878 & 2.174 & 0.650 \\
\hline 22 & -0.442 & 0.893 & 2.710 & 0.500 \\
\hline 23 & -1.836 & 1.779 & 2.301 & 0.200 \\
\hline 24 & -1.435 & 1.698 & 2.304 & 0.275 \\
\hline 25 & -1.836 & 1.779 & 2.807 & 0.300 \\
\hline 26 & -1.802 & 1.682 & 2.753 & 0.280 \\
\hline 27 & -1.396 & 1.584 & 2.753 & 0.365 \\
\hline 28 & -1.791 & 1.628 & 2.753 & 0.260 \\
\hline 29 & 1.000 & 0.969 & 2.446 & 0.850 \\
\hline 30 & -0.271 & 1.175 & 2.446 & 0.550 \\
\hline 31 & -0.271 & 1.133 & 2.693 & 0.585 \\
\hline 32 & 0.645 & 0.860 & 2.287 & 0.700 \\
\hline 33 & -1.013 & 0.948 & 2.287 & 0.305 \\
\hline 34 & -1.013 & 0.860 & 2.693 & 0.345 \\
\hline 35 & 0.901 & 1.217 & 2.662 & 0.910 \\
\hline 36 & -0.753 & 1.012 & 2.512 & 0.400 \\
\hline 37 & 1.198 & 0.937 & 2.538 & 0.920 \\
\hline 38 & -0.753 & 1.002 & 2.693 & 0.440 \\
\hline 39 & -0.753 & 0.964 & 2.325 & 0.405 \\
\hline
\end{tabular}




\begin{tabular}{|c|c|c|c|c|}
\hline 40 & 1.079 & 0.895 & 2.325 & 0.820 \\
\hline 41 & -0.753 & 0.960 & 2.708 & 0.435 \\
\hline 42 & -1.210 & 1.000 & 2.570 & 0.275 \\
\hline 43 & -1.959 & 1.065 & 2.563 & 0.150 \\
\hline 44 & -2.395 & 1.000 & 2.702 & 0.050 \\
\hline 45 & 1.000 & 0.895 & 2.500 & 0.900 \\
\hline 46 & 0.610 & 0.800 & 2.200 & 0.670 \\
\hline 47 & 0.350 & 0.680 & 2.280 & 0.570 \\
\hline 48 & 0.530 & 0.720 & 2.550 & 0.710 \\
\hline 49 & 0.280 & 0.650 & 2.720 & 0.645 \\
\hline 50 & 0.350 & 0.620 & 2.620 & 0.630 \\
\hline $51^{t}$ & -1.450 & 0.895 & 2.820 & 0.250 \\
\hline $52^{t}$ & -1.630 & 0.780 & 2.780 & 0.175 \\
\hline $53^{t}$ & -0.753 & 1.619 & 2.350 & 0.500 \\
\hline $54^{\mathrm{t}}$ & 0.345 & 0.878 & 2.700 & 0.715 \\
\hline $55^{\mathrm{t}}$ & -1.435 & 1.698 & 2.062 & 0.250 \\
\hline $56^{\mathrm{t}}$ & -1.396 & 1.671 & 2.810 & 0.385 \\
\hline $57^{\mathrm{t}}$ & -0.710 & 1.000 & 2.534 & 0.415 \\
\hline $58^{\mathrm{t}}$ & 0.921 & 0.895 & 2.483 & 0.810 \\
\hline $59^{t}$ & -0.753 & 0.978 & 2.445 & 0.385 \\
\hline $60^{t}$ & -0.753 & 0.895 & 2.719 & 0.480 \\
\hline $61^{\mathrm{t}}$ & -0.753 & 1.240 & 2.626 & 0.510 \\
\hline $62^{\mathrm{t}}$ & -0.815 & 1.228 & 2.626 & 0.505 \\
\hline $63^{t}$ & -2.395 & 1.228 & 2.736 & 0.100 \\
\hline
\end{tabular}

" $\mathrm{t}$ " is representing the testing set. 


\section{Reference}

Ay, M., Kisi, O., 2014. Modelling of chemical oxygen demand by using ANNs, ANFIS and k-means clustering techniques. Journal of Hydrology 511, 279-289.

Azadeh. A., Gaeini. Z., Haghighi. S., Nasirian. B., 2016. A unique adaptive neuro fuzzy inference system for optimum decision making process in a natural gas transmission unit. Journal of Natural Gas Science and Engineering. 34, 472-485.

Azadeh. A., Saberi. M., Rouzbahman. M., Saberi. Z., 2013. An intelligent algorithm for performance evaluation of job stress and HSE factors in petrochemical plants with noise and uncertainty. Journal of Loss Prevention in the Process Industries 26(1), $140-152$.

Azadeh, A., Ghaderi, S.F., Sohrabkhani, S., 2008. Annual electricity consumption forecasting by neural network in high energy consuming industrial sectors. Energy Conversion and Management 49 (8), 2272-2278.

Borysiewicz, M., Kowal , K., Potempski, S., 2015. An application of the value tree analysis methodology within the integrated risk informed decision making for the nuclear facilities. Reliability Engineering System Safety 139, 113-119.

Broomhead, D., Lowe, D., 1988. Multivariable functional interpolation and adaptive networks. Complex System 2(6), 321-355.

Christ, M.C., Parvathi, R.M.C., 2011. Segmentation of medical image using clustering and watershed algorithm. American Journal of Applied Sciences 8 (12), 13491352.

Golmohammadi, D., 2011. Neural network application for fuzzy multi-criteria decision making. International Journal of Production Economics. 131, 490-504. 
Guo, L., Gao, J., Yang, J., Kang, J., 2009. Criticality evaluation of petrochemical equipment based on fuzzy comprehensive evaluation and a BP neural network. Journal of Loss Prevention in the Process Industries 22, 469-476.

Hartigan, J.A., Wong, M.A., 1979. Algorithm AS 136: A K-means clustering algorithm. Journal of the Royal Statistical Society. Series C (Applied Statistics) 28 (1), 100108.

Makridaks, S., 2017. The forthcoming Artificial Intelligence (AI) revolution: Its impacts on society and firms. Futures 90, 46-60.

Özkan, G., İnal, M., 2014. Comparison of neural network application for fuzzy and ANFIS approaches for multi-criteria decision making problems. Applied Soft Computing24, 232-238.

Pitrowski, A.P., Napiorkowski, M.J., Napiorkowski, J.J., Osuch, M., 2015. Comparing various artificial neural network types for water temperature prediction in rivers. Journal of Hydrology 529, 302-315.

Seetha, M., Sunitha, K.V.N., Devi, M., 2012. Performance assessment of neural network and K-nearest neighbour classification with random subwindows. International Journal of Machine Learning and Computing 2 (6), 844-847.

Sridevi, S., Nirmala, S., 2016. ANFIS based decision support system for prenatal detection of Truncus Arteriosus congenital heart defect. Applied Soft Computing $46,577-587$.

Vanem, E., Antão, P., Østvik, I., Comas, F., 2008, Analysing the risk of LNG carrier operations, Reliability Engineering and System Safety, 93 (9), 1328-1344.

Wei, L.Y., 2016. A hybrid ANFIS model based on empirical mode decomposition for stock time series forecasting. Applied Soft Computing 42, 368-376. 
Xin, J., Chen, S., 2016. Bus dwell time prediction based on KNN. Procedia Engineering $137,283-288$.

Yang, Y., Chen, Y.H., Wang, Y., Li, C., Li, L., 2016. Modelling a combined method based on ANFIS and neural network improved by DE algorithm: A case study for short-term electricity demand forecasting. Applied Soft Computing 49, 663-675.

Zhang, R., Tan, H., 2018. An integrated human reliability based decision pool generating and decision making method for power supply system in LNG terminal. Safety Science 101, 86-97. 